\title{
Variation of Velocity and Potential of Orbit Bounded Electron with Free Electron Beam
}

\author{
B. Koirala
}

\section{ABSTRACT}

\begin{abstract}
The disturbance of free electron to bounded electron (columb potential) with different free velocity cause changes in potential and velocity of bounded electron when passes nearby. This disturbance increases and decrease both the velocity and potential of the bounded electron. The velocity changes due to repulsion between charges and this repulsion change the distance separation between bounded electron and positively charge electron. After passing away the free electron nearby the bounded electron both velocity and potential are regained due to self-energy of bounded electron. The change in potential is symmetric at the instant because the repulsion distance between bounded and free electron is changed symmetrically.
\end{abstract}

Keywords: free and bounded electron, potential,repulsion, self-energy, velocity.
Published Online: September 03, 2021

ISSN: $2684-4451$

DOI : 10.24018/ejphysics.2021.3.5.85

\section{B. Koirala*}

Department of Physics, Patan Multiple Campus, Tribhuvan University, Lalitpur44700, Nepal.

(e-mail: koiralabibek456@gmail.com)

*Corresponding Author

\section{INTRODUCTION}

Goldstein in 1886 shows the presence of discovery of new radiations using gas discharge method and this discovery subatomic particle had a charge with equal in magnitude but opposite in sign. Identification of electrons was done in 1900 by Thomson. In between, 1906-1914 Millikan found the charge on the electron is about $-1.6 \times 10^{-19} \mathrm{C}$ which is acceptable to present. Moreover, the mass of the electron was determined by Thomson using $\frac{e}{m_{e}}$ ratio as, $9.1 .94 \times 10^{-31} \mathrm{~kg}$.

Thomson also gives a model of the atom by scattering using $\alpha$-particles, experimentally. This experiment concludes atoms have larger space and on the basis of this experiment, Rutherford calculated the radius of atom about $10^{-10} \mathrm{~m}$ and nucleus are about $10^{-15} \mathrm{~m}$. He also purposed, most of the mass of the atom is concentrated at positive particle charge in a small region, which Rutherford called the nucleus, and round these electrons are moving with a very high-speed particular orbit. The high-speed electron around the nucleus is held by electrostatic forces of attraction between them.

Neils Bohr in 1913 is the first person to explain hydrogen atom structure and its spectrum. On the basis of electron move in a circular path at fixed radius and energy, energy of an electron is no change in orbit time. Atoms radiate or absorbed frequency of radiation when the transition occurs between two stationary states as $v=\frac{\Delta E}{h}=\frac{E_{2}-E_{1}}{h}$, where $E_{1}$ and $E_{2}$ are the energies of the lower and higher allowed energy states, respectively. The angular momentum of an electron in a fixed orbit is expressed as $m_{e} v r=n \cdot \frac{h}{2 \pi}, n=1$, $2,3 \ldots$ here $\mathrm{n}$ is integral numbers. The radius of this stationary orbit is determined by $r_{n}=n^{2} a_{0}$ where $a_{0}=52.9 \mathrm{pm}$ is Bohr orbit radius. The energy is expressed as $E_{n}=$
$-R_{H}\left(\frac{1}{n^{2}}\right), n=1,2,3, \ldots \ldots$, where $\mathrm{R}_{\mathrm{H}}$ is called Rydberg constant and its value is $2.18 \times 10^{-18} \mathrm{~J}$.

\section{REVIEW}

Rutherford gives a planetary model of a proton-electron atom and besides this, he also gives the physical meaning of Coulomb interaction in a vacuum as well as in the magnetic field. The vacuum velocity of the bounded electron at a distance in orbit is approximately equal to the velocity of light. The velocity of the electron in the orbit consists of two components (mechanical motion and photon-like motion). The velocity of the bounded electron is variable in the orbit depend upon the radius or distance from the nucleus [1]. Quantum chemistry of weakly bound shows the stability of neutral molecules using electronic structure theory. Some examples like the electron binding energies in the case of fluorine are $3.4 \mathrm{eV}$ and chlorine $3.6 \mathrm{eV}$ show that one is less or greater by $0.1 \mathrm{eV}$. The binding depends upon less the distance separation between two species at short ranges [2].

The development of theoretical modeling and simulation make easy to understand the characteristic of the bounded electron using the different theory of quantum machines and software. These are based on the charge, separation of charge, spin, external field, etc. are the basic parameters used to calculate the energies, density of state, molecular structure, and so on [3]. In some atoms, electrons are bounded loosely while in some atoms electrons are bounded strongly. This property of electrons is also observed in the material which they formed. Based on this bounded nature different theories are used to calculate the energy band structure using tightbinding models. This theory has different uses in material science with new development [4]. 
The electron particle is moving about the atomic nucleus along a circular orbit $n$ at a distance has radius $r_{e}=e^{2} / m c^{2}$. Using Maxwell equation authors derived the electron transition between neighboring quantum as $\Delta t=2 \pi \frac{n^{3} h^{3}}{m e^{4}}$, more detail in [5], here energy $\Delta \mathrm{E}$ during the transition and this is fit to Heisenberg Principle as $\Delta E \Delta t=h$ [6], [7]. An experiment shows that atomic electrons around the nucleus of the target material follow the motion of the recoil nucleus and at a certain instant the electrons are catch up and results in ionization or excitation of the atoms during scattering. This effect was taken into account by Migdal's approach, in this approximation also shows energy-momentum conservation and probability conservation [8].

Analytical and numerical analysis shows a specific energy dispersion law of free electrons in narrow bands leads to the possibility of pair formation induced by Coulomb repulsion between electrons. The Coulomb electron pairs exist even at finite electron density one can study the detail in the superconductivity field [9]. The energy difference between the singlet and triplet, $\Delta E=E_{S}-E_{T}=\Delta E=2 K_{a b}-\frac{(\Delta e)^{2}}{U}$, here $K_{a b}$ is the exchange repulsion between the orbitals $\chi_{a}$ and $\chi_{b}$. If $K_{a b}>0$, the triplet state is more stable than the singlet state. Also give them information about degenerate and nondegenerate which is dependent upon $\Delta e$ where energy difference of molecular orbital [10], [11]. Hartree Fock's theory explains more detail about the effect of the electron in many body problem. The orbitals excited state mean field theory can also optimized with the help of self-consistent oneelectron equation in electron-electron repulsion is accounted [12]. Electron bounded around the nucleus with finite temperature, radius, cross-section area within medium surrounding around it. the electron gets ejected after receiving energy greater than the threshold [13], [14].

\section{METHODS AND MATERIALS}

Let us consider $r_{n}$ is the radius for nth orbit where electron's has velocity $v_{n}$, the electron revolving around the post charge nucleus is given by the ides of Newtonian mechanics. That means an object goes in a circular path around the center due to centrifugal and centripetal force. This idea was implemented by Bohr's as the electron revolved around the nucleus due to electrostatic pull between positively charged nucleus at center and negatively charged particle around it. The pull force is centripetal that causes the electron to move in a circle around the nucleus and this is identical with electrostatic force, therefore we can write an equation as:

$$
\frac{m_{e} v_{n}^{2}}{r_{n}}=\frac{1}{4 \pi \epsilon_{0}} \frac{e^{2}}{r_{n}^{2}}
$$

Here, $e$ is the charge of the electron, also using the first quantization condition we can obtain the velocity and radius of the electron as:

$$
\begin{aligned}
& v_{n}=\frac{1}{4 \pi \epsilon_{0}} \frac{e^{2}}{\hbar} \frac{1}{n} \\
& r_{n}=\frac{4 \pi \epsilon_{0} \hbar^{2} n^{2}}{m_{e} e^{2}}
\end{aligned}
$$

Since an electron has speed in the orbital, therefore, the total energy $\left(K_{n}\right)$ is obtained for considering nth orbit as:

$$
K_{n}=\frac{1}{2} m_{e} v_{n}^{2}=\frac{1}{32 \pi^{2} \epsilon_{0}^{2}} \frac{m_{e} e^{4}}{\hbar^{2}} \frac{1}{n^{2}}
$$

Also, the electrostatic potential energy of interaction between two charges on the nth orbit of the atom is obtained as:

$$
U_{n}=-\frac{e^{2}}{4 \pi \epsilon_{0} r_{n}}=-\frac{1}{16 \pi^{2} \epsilon_{0}^{2}} \frac{m_{e} e^{4}}{\hbar^{2}} \frac{1}{n^{2}}
$$

Now total energy of the electron in orbit is:

$$
E_{n}=K_{n}+U_{n}=-\frac{1}{32 \pi^{2} \epsilon_{0}^{2}} \frac{m_{e} e^{4}}{\hbar^{2}} \frac{1}{n^{2}}
$$

This is the energy of the electron in the orbit of the atom. This total energy is also called the binding energy of an electron in the orbit if the incidence energy of a free electron is greater than this bounded electron then the bounded electron becomes free. But when the electron is nearby the orbit bounded electron the repulsion between them takes place and the path of both electrons gets at this instant changes as shown below in Fig. 1.

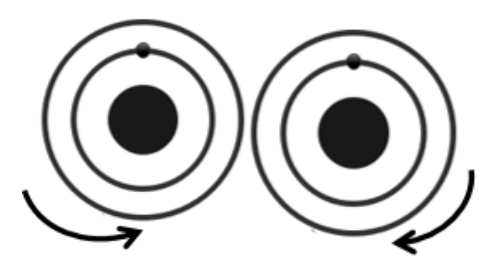

Fig.1. Revolving of the electron in orbit either clockwise or anticlockwise.

Fig. 1 shows that the velocity of an electron in orbit is either clockwise or anticlockwise this is an important visualization to calculate the change in velocity of the electron when the free electron beam passes nearby the bounded electron. The bounded electron gets deflected as shown in Fig. 2 for more detail. The velocity of a bounded electron goes decreases when a free electron passes nearby by a bounded electron revolving in the opposite direction of a free electron. On the other hand, the velocity of a bounded electron goes increases, when a free electron passes a nearby bounded electron revolving in the same direction of a free electron. The center black is the nucleus of the Hydrogen atom since we are considering a one-electron system and the small black dot is an electron revolving around the nucleus. The arrow gives the direction of an electron revolving around the revolving of the electron is due to relation (1) and total energy is given by (6).

Fig. 2 shows the formation of well-type orbit and velocity change of bounded electron in orbit of an atom. Fig. shows that potential well is formed as potential depth that is wellformed towards the nucleus of an atom because free electron beam passes up a nearby bounded electron and due to repulsion bounded and free-electron goes deviation from its original path (orbit rotation). Similarly up shape potential well is formed when a free electron beam passes from the downside of the bounded electron. This cause change in 
velocity and potential of a bounded electron as described below.

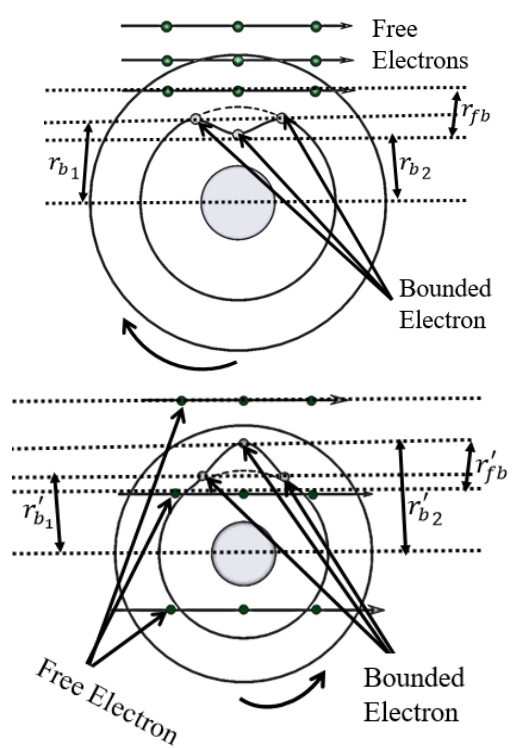

Fig. 2. Change in path of free and bounded electron due to repulsion (both condition presence of free electron nearby and absence).

\section{A. Change in Velocity of Bounded Electron due to Free Beam Electron}

As we discussed above the velocity of bounded electron in orbit is given in equation (2) which has no any external effect. Since we are considering the repulsion of between free and bounded electron and direction of beam incidence and revolution of bounded electron direction. Therefore, the velocity goes effect, let $v_{f}$ the velocity of free beam electron incidence (Fig. 2) and $v_{b}$ is the velocity of the electron clockwise. This show both direction of the bounded and freeelectron is same and hence resultant velocity is given $v_{c}$ and given by:

$$
v_{f}=\sqrt{\frac{2 K_{f}}{m_{e}}}
$$

Here $v_{f}$ is the velocity of free electron and $m_{e}$ mass of the electron, $K_{f}$ is the energy of free electron:

$$
v_{c}=v_{f}+v_{b}=\frac{1}{4 \pi \epsilon_{0}} \frac{e^{2}}{\hbar} \frac{1}{n}+\sqrt{\frac{2 K_{f}}{m_{e}}}
$$

or in a.u. equation (7), become $v_{c}=\frac{1}{n}+\sqrt{2 K_{f}}$ as, $\frac{1}{4 \pi \epsilon_{0}}=$ $1, e=\hbar=m_{e}$.

Note Energy of incidence electron is limited by the threshold energy of the bounded electron. Here $v_{b}$ is the velocity of the bounded electron. The resultant velocity with kinetics energy for other orbital is listed in the table below.

TABLE I: RESULTANT VELOCITY OF ELECTRON IN ORBIT AT AN INSTANT WHEN GOES FREE ELECTRON PASSES NEARBY WITH 1A.U. ENERGY UP \begin{tabular}{ccc}
\multicolumn{3}{c}{ BOUNDED ELECTRON IN THE SAME DIRECTION } \\
\hline S.N. & $\begin{array}{c}\text { Orbit of } \\
\text { electron }(n)\end{array}$ & $\begin{array}{c}\text { Resultant Velocity } \\
(\text { a. } u .)\end{array}$ \\
\hline 1 & 1 & 2.41 \\
2 & 2 & 1.91 \\
3 & 3 & 1.75 \\
4 & 4 & 1.66 \\
\hline
\end{tabular}
From Fig. 2, the graph shows the bounded electron velocity is anticlockwise and opposite to the free electron beam therefore the resultant velocity of orbit electron goes decreases and obtain as similar of equation (7),

$$
v_{c}^{\prime}=v_{b}-v_{f}=\sqrt{\frac{2 K_{f}}{m}}-\frac{1}{4 \pi \epsilon_{0}} \frac{e^{2}}{\hbar} \frac{1}{n}
$$

This relation shows how the velocity goes decrease, this decreasing velocity is due to the repulsive nature of the electron (free and bounded electron). The resultant velocity of an electron at an instant with a different orbit is listed in the table below.

TABLE II: RESULTANT VELOCITY OF ELECTRON IN ORBIT AT AN INSTANT When Goes Free Electron PASSES NEARby up Bounded EleCtron IN

\begin{tabular}{ccc}
\multicolumn{3}{c}{ OPPOSITE DIRECTION } \\
\hline \multirow{2}{*}{ S.N. } & $\begin{array}{c}\text { Orbit of } \\
\text { electron }(n)\end{array}$ & $\begin{array}{c}\text { Resultant velocity } \\
(\text { a.u } u\end{array}$ \\
\hline 1 & 1 & 0.41 \\
2 & 2 & 0.91 \\
3 & 3 & 1.08 \\
4 & 4 & 1.16 \\
\hline
\end{tabular}

\section{B. Variation of Bounded Potential due to Free Electron}

The representation of well type shape in orbit is due to free electron, when passes nearby target or obit electron either from up or down are represent in Fig. 2. Fig. 2 represents when a free electron passes from a nearby bounded electron both electrons go on repulsion but here we consider the repulsion effect on bounded electron only. Due to repulsion, the good type path is formed and in this path, the electron goes to closure to the nucleus therefore the Hamiltonian of this system goes changes. That is system goes under perturbation due to free beam electron, the potential goes to change, this means the potential goes decrease as the velocity of the electron at this point goes decrease as they are related by:

$$
U_{n}=-\frac{e^{2}}{4 \pi \epsilon_{0} r_{n}}=\frac{1}{16 \pi^{2} \epsilon_{0}^{2}} \frac{m_{e} e^{4}}{\hbar^{2}} \frac{1}{n^{2}}=v_{n} \frac{m_{e} e^{2}}{4 \pi \epsilon_{0} \hbar n}
$$

As $v_{n}=\frac{1}{4 \pi \epsilon_{0}} \frac{e^{2}}{\hbar} \frac{1}{n}$, On using the relation developed above for resultant velocity we have the potential at the instant is obtained as:

$U_{c}=-\frac{e^{2}}{4 \pi \epsilon_{0} r_{b}}=-\left(\frac{1}{4 \pi \epsilon_{0}} \frac{e^{2}}{\hbar} \frac{1}{n}+\sqrt{\frac{2 K_{f}}{m_{e}}}\right) \frac{m_{e} e^{2}}{4 \pi \epsilon_{0} \hbar n}, r_{n}>r_{b}(10)$

This relation shows that potential at the point $r_{b}$ is higher than $r_{n}$, this is because of the repulsion between the electrons (free and bounded). Hence potential at $r_{b}$ is higher than $r_{n}$ and relation (9) show velocity of the electron at point should high than $r_{n}$ orbit radius. This is for the electron revolution around the nucleus system.

$$
U_{c}^{\prime}=-\frac{e^{2}}{4 \pi \epsilon_{0} r_{b}^{\prime}}=-\left(\sqrt{\frac{2 K_{f}}{m}}-\frac{1}{4 \pi \epsilon_{0}} \frac{e^{2}}{\hbar} \frac{1}{n}\right) \frac{m_{e} e^{2}}{4 \pi \epsilon_{0} \hbar n}
$$


This relation shows that potential at a point $r_{b}^{\prime}$ which is less than $r_{n}$ this is because of the repulsion between the electrons (free and bounded) for down level and electron get push up more detail in Fig. 2. This shows that potential at $r_{b}^{\prime}$ is less than $r_{n}$ and hence the velocity of the electron at point should be less than $r_{n}$ orbit radius.

\section{RESUlTS AND DisCUSSION}

\section{A. Variation of Velocity Repulsion between Free and Bounded Electron}

The graph below represented the resultant velocity of bounded orbit electron when free electron passes nearby bounded electron for two cases. Case I, incidence beam of a free electron and bounded electron are in the same direction (free-electron linear velocity and translation velocity of bounded electron in orbit), this show the repulsion increase the bounded velocity of the electron, but with increasing the orbit the velocity for bounded electron goes decrease, this is because of conservation of energy (kinetic energy + potential energy) of the bounded electron. Therefore, due to the distance separation between columb charges increase. This separation distance bounded electron loosely and the total energy of the electron is less, Fig. 3 represents the velocity of a bounded electron with increasing quantum number with the incidence of a free electron in the same direction.
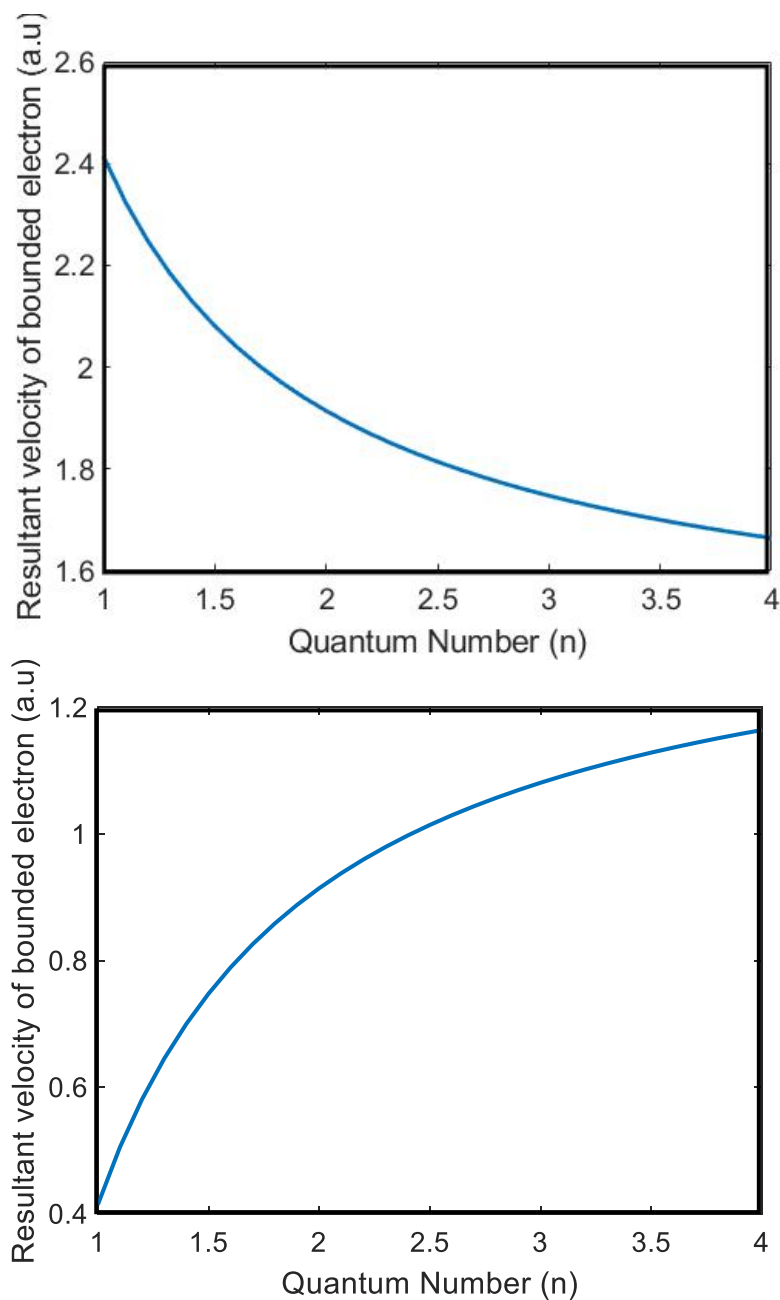

Fig. 3. Velocity with the same and opposite direction.
Case II, incidence beam of a free electron and bounded electron are in opposite direction (free-electron linear velocity and translation velocity of bounded electron in orbit), this also show the repulsion increase bounded velocity of electron which resists the bounded electron velocity and velocity goes decrease but with increasing orbit the velocity goes increase. This is because of the conservation of energy (total kinetic energy + total potential energy). This is like Zener diode as a voltage regulating circuit.

\section{B. Comparison Bounded Electron Velocity with Free Electron with Direction}

Since repulsion takes place in both cases either electron or free-electron are in the same direction or opposite direction. But the velocity with the same direction goes increases while with opposite direction goes decrease in the same orbit. If we increase in higher-order orbit (increase the quantum number (n)) the velocity in the orbit has the same effect with incidence free electron but the resultant velocity for lower quantum number is greater than a higher quantum number, for the same direction; while the resultant velocity is opposite that is resultant velocity is high for higher quantum number and lower for the lower quantum number for the opposite direction. This is because the total energy of orbit flows the rule of conserved since the atom is not going ionized in this work. Therefore, for the quantization of energy the velocity changes with the orbit of an atom which is repressed below in Fig. 4.

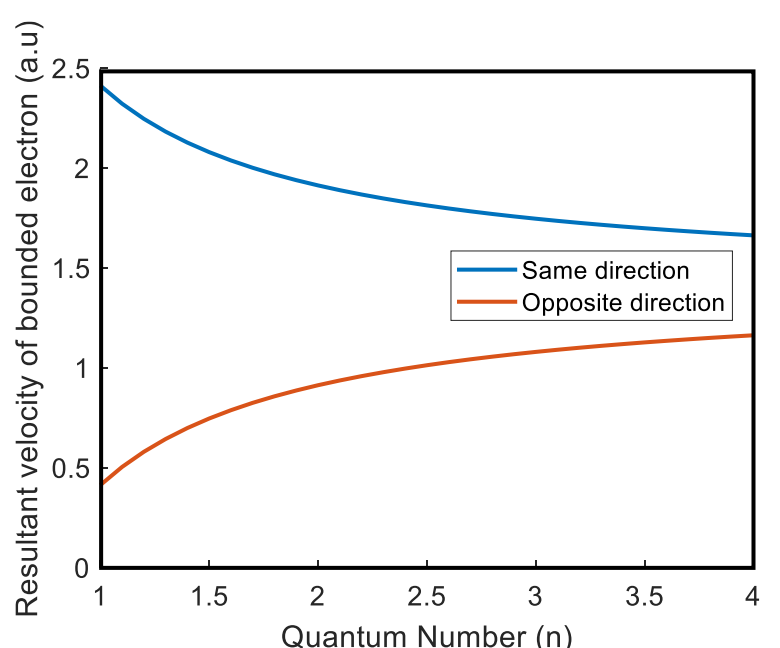

Fig.4. Comparison of bounded electron velocity (rotational) with a free electron (linear)

\section{Potential with the Same Direction of Electron}

Fig. 2 shows the free electron passes above the bounded electron and orbit shape at this instant is formed type as well. Since the distance between the free electron and fixed common nucleus from Fig. 2 is $\left(r_{b_{2}}+r_{f b}\right)$ and distance separation at which repulsion between bounded and freeelectron is high is $r_{f b}$. At this point $r_{b_{2}}<r_{b_{1}}$ therefore potential at this point is higher when an electron passes a nearby bounded electron at this point. This is because potential depend upon inversely with distance. The potential goes decrease with the increasing distance shown in Fig. 5, also with the increase quantum number potential goes decrease. This potential is the case when the direction of a free electron and bounded electron are the same. 
Fig. 2 shown the free electron passes below the bounded electron and orbit shape at this instant is formed as peak well type. The distance between free the electron with the fixed common nucleus are varied, but we consider some fixed distance to study the potential when a free electron passes below the bounded electron from Fig. 2. The minimum distance separation between the bounded electron due to which bounded electron repulse by the free-electron beam is $\left(r_{b_{2}}^{\prime}\right)$. This distance is greater than bounded electrons which form peak well type orbital due to repulsion that is $r_{b_{2}}^{\prime}>r_{b_{1}}^{\prime}$ and at this point potential of the bounded electron at position $r_{b_{1}}^{\prime}$ is higher than bounded repulsed electron at position $r_{b_{2}}^{\prime}$ because potential depend upon inversely with distance. The nature of potential when a free electron passes from below of bounded electron are observed in Fig. 5. This potential is the case when the direction of a free electron and bounded electron are opposite.
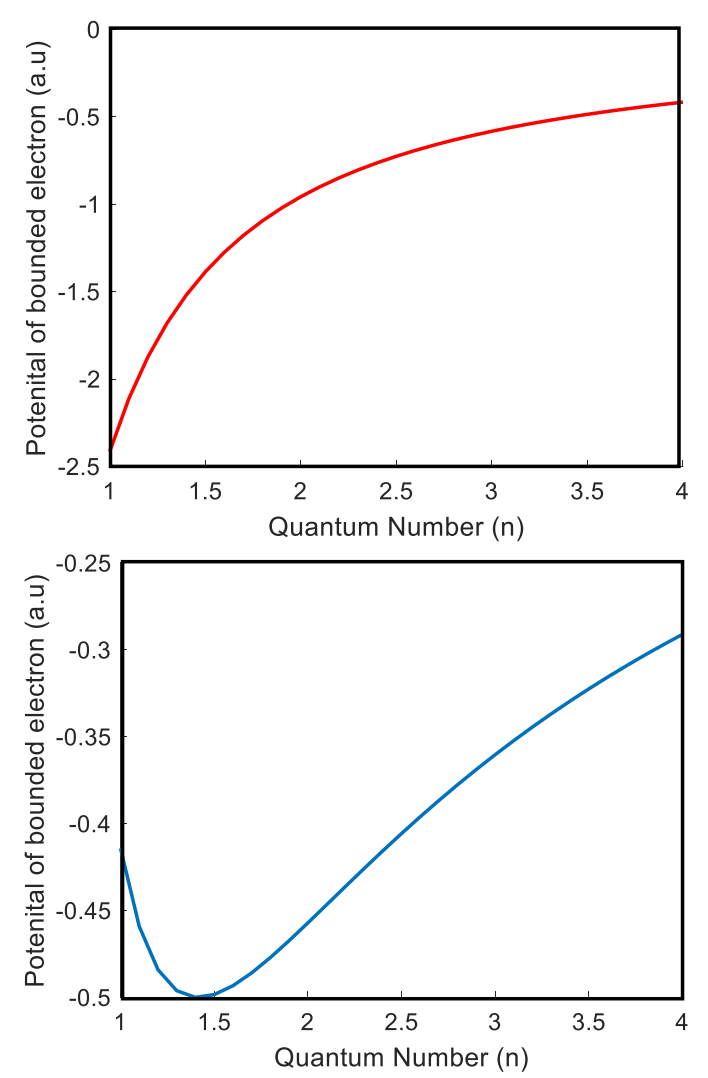

Fig. 5. Potential same and opposite direction.

\section{Comparison of Potential}

The potential nature with the same and opposite direction of the free and bounded electron is described in Fig. 5. The comparative study shows that the same orbit and same bounded electron with free-electron when passes nearby them are given below in Fig. 6. When a free electron passes nearby opposite direction (revolution) of the bounded electron the velocity goes decrease and potential is low $r_{b_{2}}^{\prime}$ because this is the maximum distance due to repulsion between free and bounded electrons when an electron passes below the bounded electron. When the direction is the same the potential is high at $r_{b_{2}}$ point this is because of the repulsion between the free and electron when the free electron passes from up of the bounded electron.
The nature of the potential for the same direction and opposite direction with different quantum numbers and the same energy of free electrons are shown in Fig. 6 below. This nature shows that the potential for the same direction of electrons potential goes decrease with increase quantum number for the same atom, while for the opposite direction of electrons the potential goes increase initially and then goes decrease for the same atom.

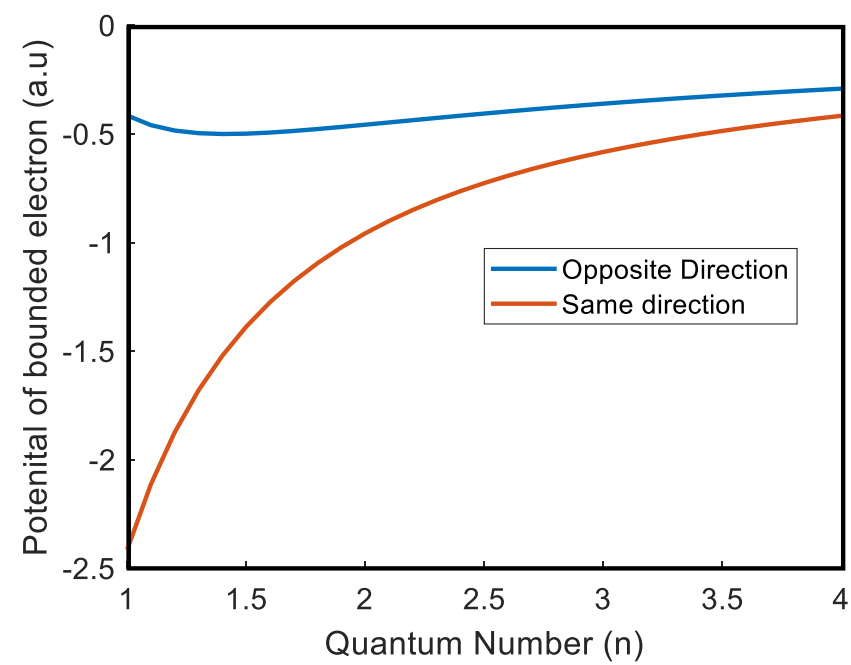

Fig. 6. Nature of Potential with direction of the free and bounded electron.

\section{CONCLUSION}

The effect of the free electron was observed on bounded electrons when the free electron passes nearby the bounded electron. The observation and mathematical mode develop shows that potential, and velocity is getting effect by free electron. The detailed description is visualized in the methodology section in Fig. 2, the velocity and potential at $r_{b_{2}}$ and $r_{b_{2}}^{\prime}$ position changes, the potential at point $r_{b_{2}}$ is maximum and velocity is minimum, when free electrons pass from upside nearby bounded electron with the same direction. Similarly, the potential at the point $r_{b_{2}}^{\prime}$ are minimum and velocity is maximum, when free electrons pass from upside nearby bounded electron with opposite direction. This maximum and minimum appear to preserve the quantization and law of conservation of energy.

Also, the velocity for the same direction goes decrease with the increasing quantum number of the same atom while increase with increasing in quantum number in opposite direction. Since velocity is dependent upon potential with the same nature potential also changes. The effect of free electron causes the perturbation on Hamiltonian of the system therefore the variation is observed as the electron passes nearby bounded electron.

\section{REFERENCES}

[1] R. Fedaruk, Two types of proton-electron atoms in a vacuum and an extremely strong magnetic field, 2021, pp. 1-2 https://arxiv.org/ftp/arxiv/papers/1008/1008.4666.pdf.

[2] J. M. Herbert, Chapter 8: The Quantum Chemistry of Loosely-Bound Electrons, Reviews in Computational Chemistry, John Wiley \& Sons, Inc., vol. 28, 2015, pp. 391-392.

[3] E. H. Lieb, Quantum Mechanics: The Stability of Matter and Quantum Electrodynamics, 2003, pp.1-2, http://arxiv.org/abs/math$\mathrm{ph} / 0401004 \mathrm{v} 1$. 
[4] F. R. Ramírez, E. F. Olmedo, G. Báez, E. Sadurní, and R.A. M. Sánchez, "Emulating tightly bound electrons in crystalline solids using mechanical waves," Scientific Reports, vol. 10, no. 10229, 2020, pp. 15. https://doi.org/10.1038/s41598-020-67108-0.

[5] S. Olszewski, "Time Intervals of the Electron Transitions between the Energy States in the Hydrogen Atom Calculated in a Non-Probabilistic Way," Journal of Modern Physics, vol.10, 2019, pp. 1522-1531. doi: 10.4236/jmp.2019.1013101.

[6] S. Olszewski, "Non-Probabilistic Approach to the Time of Energy Emission in Small Quantum Systems," Journal of Modern Physics, vol.6, 2015, pp. 1277-1288. doi: 10.4236/jmp.2015.69133.

[7] H. Lass, "Vector and Tensor Analysis," American Journal of Physics, vol. 18, no. 583, 1950, 3-5. https://doi.org/10.1119/1.1932684

[8] M. Ibe, W. Nakano, Y. Shoji and K. Suzuki1, Migdal Effect in Dark Matter Direct Detection Experiments, IPMU17-0100, 2020, 1-2. https://arxiv.org/pdf/1707.07258.pdf.

[9] K. M. Frahmand and D. L. Shepelyansky, "Electron pairing by Coulomb repulsion in narrow band structures," Physical Review Research, vol.2, no.023354, 2020, pp.1-2. doi.org\%2F10.1103\%2FPhysRevResearch.2.023354.

[10] P.J. Hay, J.C. Thibeault and R. Hoffmann, "Orbital interactions in metal dimer complexes," Journal of American Chemical Society, vol 97, 1975, pp. 4884-4899.

[11] P. A. Maggard, X. Cheng, S. Deng and M. H. Whangbo, "Physical Properties of Molecules and Condensed Materials Governed by Onsite Repulsion, Spin-Orbit Coupling and Polarizability of Their Constituent Atoms," Molecules, vol. 25, no. 867, 2020, pp. 1-14 doi: $10.3390 /$ molecules 25040867 .

[12] T. S. Hardikar and E. Neuscamman, A Self Consistent Field Formulation of Excited State Mean Field Theory, 2020, pp.1-10. https://escholarship.org/content/qt223816p1/qt223816p1_noSplash_f6 28137268abe8320148b37b2645d545.pdf.

[13] B. Karki, S. H. Dhobi, K. Ghimire, D. Kharel, S. Ghimire, "Temperature of Electron Inside and Outside of Atom," Technology Reports of Kansai University, vol.63, no.3, 2021, pp.7483-7491.

[14] S. H. Dhobi, C.B.T. Lama, MD.J. Rangrej, T. R. Karki, Y. Limbu, S. Humagain, "Numerical Analysis of Photon and Electron Size, and Verifying One to One Correspond Rule of Einstein Photoelectric Effect," International Journal of Scientific \& Engineering Researchvol.10, no.11, 2019, pp.838-841. 